\title{
The light fantastic
}

\section{A decade ago,} holographic

\section{systems promised}

to revolutionize data storage. The early hype may have evaporated, but the technology quietly progressed, and working devices are now on the market. Mark Haw reports.

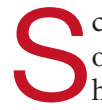
cience is suffering from information overload. Astronomers struggle with huge image files from their telescopes; high-energy physicists are bombarded with numbers; and a new kind of scientist — the bioinformatician — has emerged over the past decade to help to make sense of vast biological databases. Some companies can now turn a profit simply by storing other peoples' data ${ }^{1}$.

Computer memory is cheap, so this data glut is of little concern to most researchers. Yet the way in which we store information literally only scratches the surface of what is possible. Humans have been recording data on surfaces since the era of clay-tablet accounting systems. The printing press, photography, magnetic tape and compact discs (CDs) have each transformed data storage. But if we want to go further, and cram more data into less space, we may have to move beyond surface technologies, and actually store information inside materials — in the form of a hologram.

Ten years ago, with magnetic-disk technology seemingly reaching its maximum potential, holographic data storage seemed destined for the centre stage. But as the capacity of magnetic disks defied expectations, interest in holographic alternatives waned, and big companies ceased their research efforts. A few smaller firms, however, as well as some academic researchers, kept the faith. Spurred on partly by the advent of digital videos and problems with archiving
Rays of hope: light-sensitive crystals that record patterns of laser light are being hailed by some as the next generation of data-storage media. feature films, commercial holographic storage options are beginning to emerge.

The potential advantages of holograms are indisputable. Opening up the inside of a material for storage, rather than just using its surface, yields huge improvements in capacity. For example, InPhase, a holographictechnology company based in Longmont, Colorado, is marketing a disk that is similar in size to a standard CD but can store 200 gigabytes - a 200-fold improvement on optical CD technology. Data can also be read more quickly from such systems. And because of the way in which information is stored, useful new types of searching technique are possible.

\section{Bright idea}

The basic technique for such storage was proposed in 1963 by Pieter van Heerden, who was working on holographic technologies at Polaroid's labs in Cambridge, Massachusetts $^{2}$. The ones and zeros that make up the data set are first split into twodimensional pages of data - lines of light and dark pixels displayed on a screen. Individual pages are then illuminated from behind with a laser, producing a projection of the page known as a data wave.

To store a page, the data wave is projected onto a block of transparent material, together with a second pattern of dark and bright pixels called the reference wave. The peaks and troughs of the two waves interfere to form a new pattern and, if the block is made from a substance that reacts to light and dark in different ways, the interference pattern is stored inside the material.

Taking a single page and turning it into a three-dimensional pattern isn't much use in itself. The trick of holography is that, by using a different reference beam for each data page, many pages can be stored in the same volume. This is possible because the angle between the data and reference waves determines how the peaks and troughs of the two beams line up, and hence dictates the interference pattern. By varying this angle for different pages, an entire data set can be stored in a single block.

In the same way that a radio can be tuned to a single frequency amid the overlapping transmissions from other stations, a third kind of light beam — the reading wave — can be used to extract individual pages from the many patterns stored in the block. Any beam directed at the block will be scattered by the patterns, but if the reading wave has the same wavelength as a particular reference wave, and is beamed into the block at the same angle, it will pick out the data page stored using that reference wave. The scattered light will be a recreation of the data page, which can be read into a computer by projecting it onto a video camera.

Van Heerden's technique is known as holographic data storage, as the method for storing the pages is similar to that used to create holograms - the different pages of data are analogous to the different views of an 


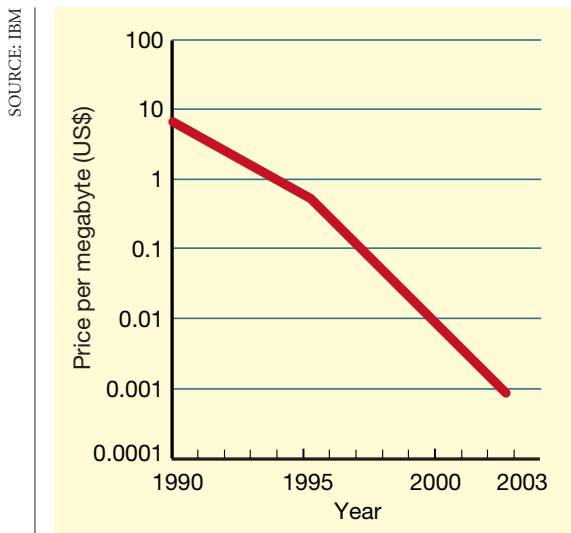

Attractive prospect: the cost of magnetic storage has fallen rapidly in recent years.

Ingenious ways of fixing the holographic memory have since been explored, and one method looks like it might be up to the job. In 1998, Karsten Buse, an optics researcher then at the California Institute of Technology in Pasadena, described how his group added manganese atoms to a lithium niobate crystal. These act as both suppliers and traps of electrons. Because the atoms hold onto their electrons strongly, a high-energy ultraviolet beam is needed to release the electrons. The electrons move in response to the interference pattern, but some are recaptured by the manganese traps. The distribution of recaptured electrons is a copy of the pattern, and is stable because reading with a lower-energy beam does not release these electrons ${ }^{6}$.

Such memories are rewritable, as the ultraviolet beam can release the trapped electrons and wash out the recorded copy of the data when this is required. The idea of using a high-energy beam to switch recording on and off, while a low-energy beam allows nondestructive reading, arose at Bell Laboratories in the 1970s (ref.7). The lasers and optical components of the time were too expensive and of insufficient quality for the technique to be feasible - but this is not the case today. Researchers are now trying to reduce the time taken to store data pages - using an affordable 1-milliwatt device, this currently takes more than ten minutes per page. Buse, now at the University of Bonn in Germany, continues to work on holographic systems, and is confident that problems such as the exposure time can be ironed out.

So are we entering the era of desktop holographic data storage? Not yet, says Hans Coufal of IBM's Almaden Research Centre in San Jose, California. "At the beginning of the 1990 s people thought we were very close to the limit of magnetic-disk capacity," he says. "IBM began seriously looking into holographic data storage." But the hard-drive capacity of a standard desktop computer has doubled annually for the past five years. There have also been difficulties in making optical components cheap enough to compete with magnetic sys- tems, whose price has consistently dropped for more than a decade (see graph, left). Coufal says this is enough to convince IBM that holographic memories are not yet ready for entry into such a fiercely competitive market.

Other researchers are more optimistic. According to Buse, the doubts are an economic rather than a technical problem - money is being channelled into tried-and-tested technologies, rather than riskier alternatives. "Industry trends are currently not in favour of holographic storage," he says. "Large funds are invested to improve the conventional technology and not to boost holography."

\section{Screen tests}

But whereas big players such as IBM have dropped out of the field, smaller companies see potential in holographic technologies. InPhase, as well as Aprilis of Maynard, Massachusetts, are marketing holographic storage systems based on photopolymers. Neither will reveal details of the polymers they use, but Aprilis claims to have reached storage capacities as high as 15 gigabytes per square centimetre, and InPhase says that Sony is interested in developing its technology. Lisa Dhar, InPhase's vice-president of media development, says that the company's 200gigabyte disk can be read at 20 megabytes per second, compared with the 5 megabytes per second that can be obtained from CDs.

The high costs involved in manufacturing the optics for the systems, together with the fact that powerful lasers are needed for recording, mean that neither company is yet aiming at the domestic market. InPhase says that it is talking to movie-industry companies such as Universal Studios and Technicolor about long-term safeguarding of video material. "There's no acceptable long-term solution," explains Dhar. "Videotape has an archive life of about ten years." The current fix is to record video onto longer-lasting photographic film. Dhar claims that InPhase's

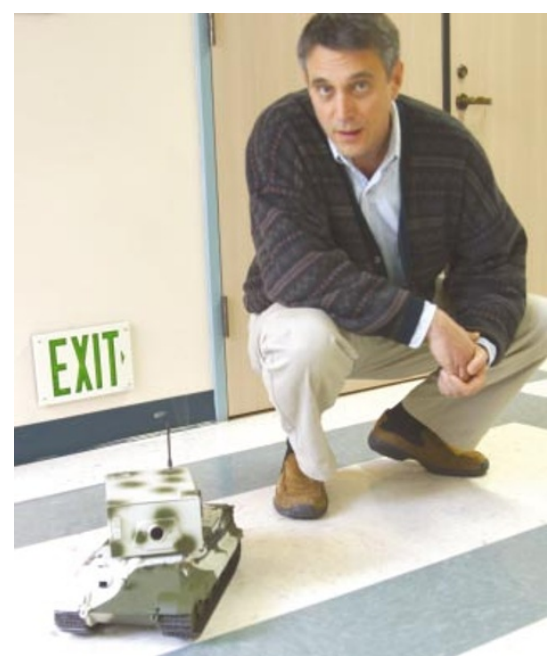

Dmitri Psaltis' robot uses a searchable memory of holographic data to help navigate its way home. holographic technology will be nearly 50 times cheaper, and that 60 minutes of uncompressed video - which would require 1,600 metres of film or 100 DVDs - could be stored on just three holographic disks.

Other possible image-archiving applications include medical imaging, oil exploration and satellite imagery. But all of the companies involved admit that it is too early to say which applications will prove profitable. "It's a bit difficult to predict how all this will shake out," says Glenn Horner, vicepresident of business development at Aprilis. "Much of the story has yet to be written."

What might eventually tip the balance towards holographic data storage is one feature that cannot be matched by other methods. Just as a data wave can be retrieved using a reference wave, a reference wave can be retrieved using a data wave. Known as content-addressable searching, this allows users to find out if a particular data page is present by illuminating a memory with the corresponding data wave. If the page is there, the original reference wave will be recreated. The method also allows for fuzzy searches - if there are several pages similar to the search page, various recreated reference beams will appear, with intensities that depend on how closely each matches the search page.

This presents exciting possibilities. In computer vision, for example, the method can be used to detect matches between images of the same scene viewed under different lighting conditions. In 1995, a team led by Dmitri Psaltis at the California Institute of Technology created a robot that navigated around a building using content-addressable searching to compare real-time camera pictures with images of known locations stored in its holographic memory ${ }^{8}$.

Work in this area continues, with some researchers linking holographic memories with artificial neural networks - computing devices based on the structure of the human brain. The combination provides rapid image recognition, and can be trained to recognize different types of object ${ }^{9}$.

After a long gestation, recent advances in materials, optical technology and laser availability are at last combining to make holographic memories a reality. Although you won't see one on your desktop just yet, even the most sceptical observers think that the technology will blossom in the long run. Eventually the elusive limit to surface storage capacity will be reached, after which the third dimension may be the only way to go.

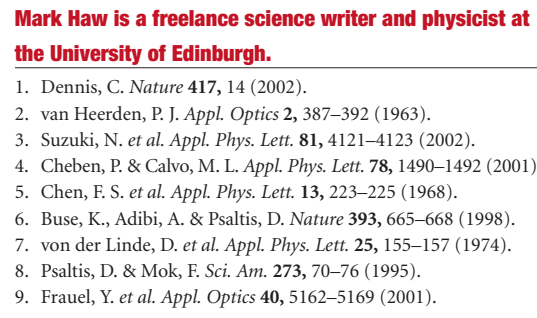

